\title{
Hearing loss in high risk neonates
}

\author{
Vaghasiya $D^{1}$, Deb $S^{2}$, Gogoi P.R ${ }^{3}$, Nongrum H.B ${ }^{4}$, Lurshay R. $M^{5}$
}

${ }^{1}$ Dr Dashrath Vaghasiya, DNB Pediatrics, Senior Resident, Department of Pediatrics, ${ }^{2}$ Dr. Santanu Deb, Senior Consultant \& HOD, Department of Pediatrics, ${ }^{3}$ Dr. Palash Ranjan Gogoi, Consultant, Department of Pediatrics, ${ }^{4}$ Dr. Henry B. Nongrum, Consultant, Department of ENT, ${ }^{5}$ Dr. Richard Mario Lurshay, DNB Pediatrics, Senior Resident, Department of Pediatrics; all authors are affiliated with Nazareth Hospital, Shillong, Meghalaya, India.

Address for Correspondence: Dr Dashrath Vaghasiya, Senior Resident, Department of Pediatrics, Nazareth Hospital, Laitumkhrah, Shillong, Meghalaya, India. Email: vaghasiyadashrath@gmail.com

\begin{abstract}
Objective: To determine the incidence and risk factors associated with hearing loss in high risk neonates. Design: Descriptive cross sectional study. Setting: Level II NICU of a tertiary care hospital. Participants: 280 high risk infants were screened. Intervention: All high-risk babies were initially screened with both transient evoked otoacoustic emissions (TOAE) and Auditory brainstem response (ABR). Outcome variables: Primary: to determine the incidence of auditory neuropathy in high risk infants; Secondary: risk factors in those babies identified with hearing loss. Results: Incidence of hearing loss was 17.8 per 1000 screened (95\% C.I is $0.24 \%$ - 3.32\%), with bilateral hearing loss seen in 14.2 per 1000 babies. Highest incidence of hearing loss was seen is in infants with intracranial haemorrhage and meningitis $(50 \%)$ followed by in decreasing orders craniofacial anomalies $(20 \%)$, intrauterine congenital infections $(11.11 \%)$, culture positive sepsis including meningitis (8.88\%), birth weight less than $1.5 \mathrm{~kg}(7.14 \%)$, preterm $<33$ weeks $(4.34 \%)$, severe birth asphyxia (4\%), use of ototoxic medications for more than 5 days $(3.03 \%)$. The risk of hearing loss is higher with higher number of risk factors. With a single risk factor being present, it is only $0.66 \%$, whereas with five risk factors, the incidence was $33.3 \%$. Conclusion: There is a high incidence of hearing loss in at-risk infants. Hearing loss is commoner when risk factors such as intracranial haemorrhage or meningitis are present and with presence of multiple risk factors. This would justify the need to routinely screen all high risk infants, so that rehabilitative measures can be initiated at the earliest.
\end{abstract}

Key words: Auditory brainstem response, Hearing, newborn, Otoacoustic emissions, Screening, Meningitis

\section{Introduction}

Hearing loss is one of the most common congenital anomalies. In the USA, the average incidence of neonatal hearing loss is $1.1 / 1,000$ infants; the rate by state varies from 0.22 to $3.61 / 1,000$ [1]. In India, incidence of hearing loss is 0.98 to 4.7 per 1000 in not at risk infants and $10.3-10.7$ per 1000 in high risk infants $[2,3]$.

Significant hearing loss present at birth is one of the most common major disabilities. It is well recognized that unidentified hearing loss can adversely affect optimal speech and language development, acquisition of literacy skills, and academic, social and emotional development. The risk is even more in a country like

Manuscript received: $24^{\text {th }}$ October 2016

Reviewed: $5^{\text {th }}$ November 2016

Author Corrected; $16^{\text {th }}$ November 2016

Accepted for Publication: $28^{\text {th }}$ November 2016
India where infrastructure is abysmally inadequate for prevention and remediation. Early detection can prevent further disabilities in speech, language and cognition in the child's development. It is established that hearing loss, if present, should be detected before the infant is 6 months old [2].

Ideally, efforts should be made to organize Universal newborn hearing screening because up to $42 \%$ of profoundly hearing impaired children may be missed using only risk-based screening. However short of universal screening, high risk screening should be mandatory [4].

A pilot study in India has shown that screening of only at risk neonates can miss detection of $70 \%$ of the newborns with hearing impairment. If the resources are 
limited, then one could focus initially on at risk neonates and gradually implement universal screening [3].

There is still a dearth of studies showing incidence of hearing loss in the North Eastern states of India. Therefore, this study aims to find the incidence of hearing loss in high risk neonates in level II care NICU, Nazareth Hospital, Shillong, in Meghalaya.

\section{Materials and Methods}

This descriptive cross sectional study was carried out in all high risk neonates born at Nazareth Hospital, Shillong, Meghalaya which has a level II NICU, with the primary aim of finding out the incidence of hearing loss in high risk neonates.

The secondary aim was to document the risk factors associated with the hearing loss. The study which was carried out for one year from December 2011 to November 2012. All neonates (0-28 days) with the following risk factors were included in the study $[5,6,7]$

A. An illness or condition requiring admission of more than 5 days to NICU.

B. Severe birth asphyxia which may include infants with APGAR score 0-4 at $1 \mathrm{~min}$ and 0-6 at $5 \mathrm{~min}$.

C. Culture positive sepsis including bacterial meningitis.

D. Ototoxic medications including, but not limited to, the aminoglycosides used for more than 5 days (eg. Gentamicin, tobramycin, streptomycin, amikacin) and loop diuretics used in combination with aminoglycosides.

E. Hyperbilirubinemia at the serum level of exchange transfusion or $2 \mathrm{mg} / \mathrm{dl}$ below that level.

F. Family history of permanent childhood sensoryneural hearing loss.

G. In utero infection such as cytomegalovirus, herpes, toxoplasmosis, or rubella (TORCH).

\section{Results}

Incidence: 280 at risk infants were screened and 5 were detected to have hearing impairment which is an incidence of 17.8 per 1000 screened (95\% C.I is $0.24 \%$ - 3.32\%). Amongst these, $4(1.42 \%)$ babies had bilateral hearing loss and 1 $(0.35 \%)$ baby had right ear hearing loss. Following flow chart shows the results as per the protocol used for study.
H. Birth weight less than 1500 grams.

I. Stigmata or other findings association with a syndrome known to include a sensory-neural and/or conductive hearing loss.

J. Craniofacial anomalies, including those with morphologic abnormalities of the pinna and ear canal.

K. Readmissions in the first month of life for all infants (NICU or well-baby) when there are conditions associated with potential hearing loss (e.g., hyperbilirubinemia requiring exchange transfusion or culture positive sepsis).

The team for the hearing screening included a Paediatrician, otorhinolaryngologist, audiologist, Staff nurses and Counsellor. In all high-risk babies, the initial screening was done by both transient evoked otoacoustic emissions (TOAE) and Auditory brainstem response $(\mathrm{ABR})$.

If infants failed the first screen, the tests in which they failed were repeated in both ears. In case the child failed ABR, the test was repeated after two months with ABR only, in both ears.

The intention here was to detect the presence of suspected auditory neuropathy and to confirm hearing loss. If a child failed ABR after two months, then behavioural observation audiometry and immittance audiometry was done before rehabilitation.

In case of a preterm or low birth weight neonate, where the ear canal was too small and the ear tip could not be snugly fitted into the canal, these neonates were asked to come for screening between 2 weeks and 1 month, till the child grew enough to enable the audiologist to fit in the ear tip.

All babies were first screened using an Intelligent Hearing Systems (IHS, Miami, Florida) Smart TrOAE which involves placing a probe into the baby's outer ear. The ABR instrument which was used is the Intelligent Hearing Systems (IHS, Miami, Florida). 


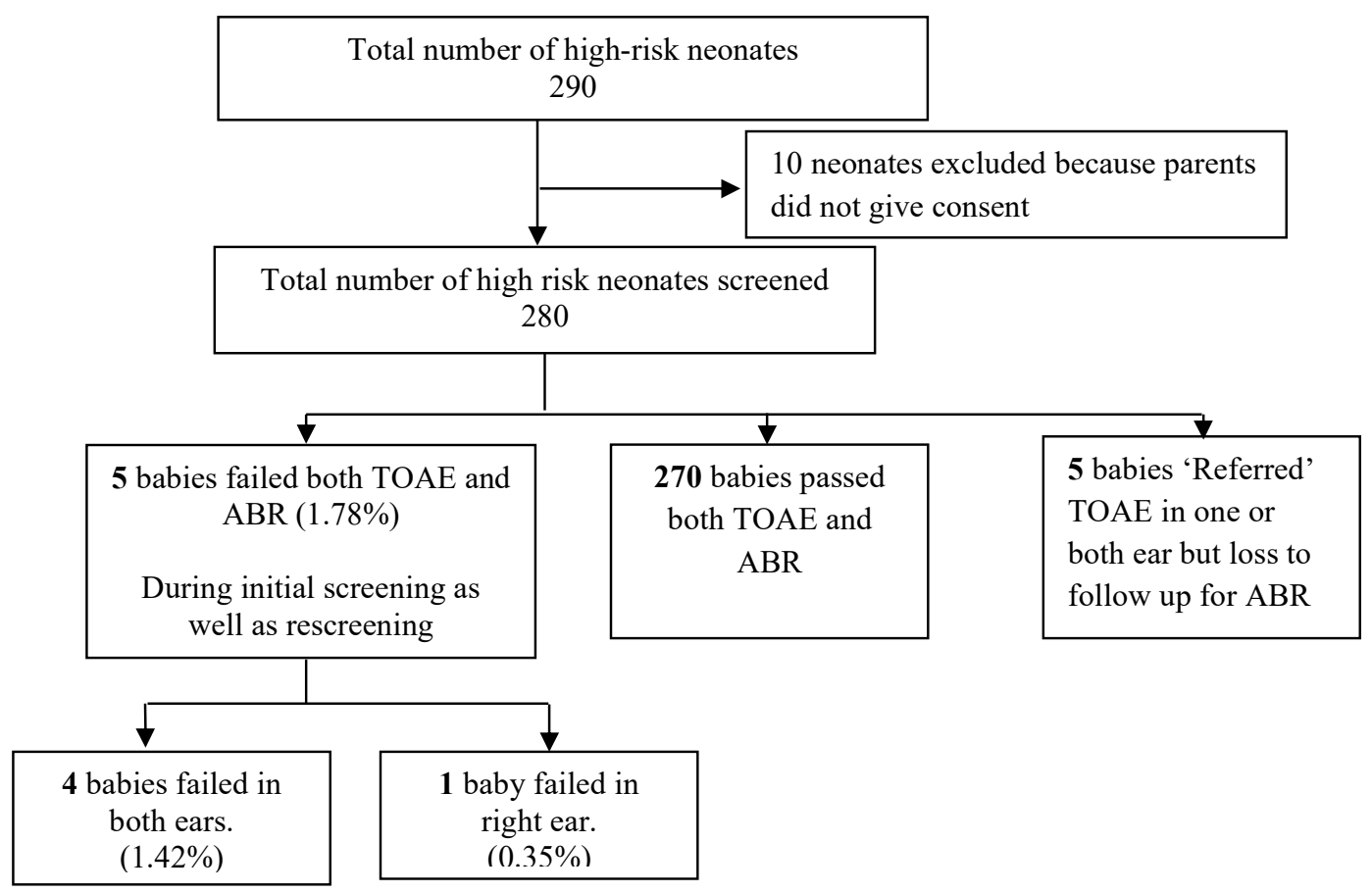

Figure-1: Flow chart of results of hearing screening in high risk neonates.

The incidences in various groups of infants with the risk factors are shown in Table 1.

\section{Risk factors}

Table-1: Incidence of hearing loss for individual risk factors.

\begin{tabular}{|c|c|c|}
\hline Risk factor & Number of babies (N=275) & Number of hearing loss (\%) \\
\hline Use of ototoxic drugs for more than 5 days & 132 & $4(3.03 \%)$ \\
\hline Stay in NICU for more than 5 days. & 128 & $4(3.12 \%)$ \\
Preterm < 33 weeks. & 23 & $1(50 \%)$ \\
Intracranial haemorrhage & 2 & $1(0.82 \%)$ \\
\hline Hyperbilirubinemia requiring exchange transfusion \\
or 2 mg/dl less than that & 121 & $4(8.88 \%)$ \\
\hline Culture positive sepsis including meningitis \\
Meningitis & 45 & $1(50 \%)$ \\
\hline Severe birth asphyxia & 4 & $1(7.14 \%)$ \\
\hline Birth weight less than 1.5 kg & 25 & $1(11.11 \%)$ \\
\hline Intrauterine congenital infections & 14 & $1(20 \%)$ \\
\hline Craniofacial anomalies & 9 & 0 \\
\hline Family history of childhood hearing loss & 8 & 0 \\
\hline Syndromes associated with hearing loss & 6 & $1(4 \%)$ \\
\hline
\end{tabular}

The highest incidence seen is in infants with intracranial haemorrhage and meningitis followed by in decreasing orders craniofacial anomalies, intrauterine congenital infections, culture positive sepsis including meningitis, birth weight less than $1.5 \mathrm{~kg}$, preterm < 33 weeks, severe birth asphyxia, use of ototoxic medications for more than 5 days. Hearing loss was not found in patients with family history of childhood hearing loss and syndromes associated with hearing loss. Craniofacial anomalies seen in patient with hearing loss was right sided facial nerve palsy with right ear cartilage malformation. An intrauterine congenital infection seen in patient with hearing loss was CMV. More than one risk factor was observed in few high risk neonates and in hearing loss babies which is shown in table 2 and 3. 
Table-2: Number of risk factors and incidence of hearing loss.

\begin{tabular}{|c|c|c|c|}
\hline Risk factor & $\begin{array}{c}\text { Number in high-risk neonates } \\
\mathbf{( \% )} \\
\mathbf{N = \mathbf { 2 8 0 }}\end{array}$ & $\begin{array}{c}\text { Number in hearing loss } \\
\text { neonates } \mathbf{( \% )} \\
\mathbf{N = 5}\end{array}$ & $\begin{array}{c}\text { Incidence of hearing loss as } \\
\text { per number of risk factors }\end{array}$ \\
\hline Single & $150(53.57 \%)$ & $1(20 \%)$ & $1 / 150=0.66 \%$ \\
\hline Two & $61(21.78 \%)$ & 0 & - \\
\hline Three & $51(18.21 \%)$ & $1(20 \%)$ & $2 / 15=13.33 \%$ \\
\hline Four & $15(5.35 \%)$ & $2(40 \%)$ & $1 / 3=33.33 \%$ \\
\hline Five & $3(1.07 \%)$ & $1(20 \%)$ & \\
\hline
\end{tabular}

Table-3: Risk factors in individual babies who had hearing loss.

\begin{tabular}{|c|c|}
\hline Case (Baby) & Risk factors \\
\hline Case no 1 & $\begin{array}{l}\text { 1. Severe birth asphyxia. } \\
\text { 2. Culture positive sepsis. } \\
\text { - Meningitis } \\
\text { 3. Use of ototoxic medications for more than } 5 \text { days. } \\
\text { 4. Hyperbilirubinemia at the level requiring exchange transfusion. } \\
\text { 5. Stay in NICU for more than } 5 \text { days. }\end{array}$ \\
\hline Case no 2 & 1. Craniofacial anomalies (Right side facial nerve palsy with right ear cartilage malformations.) \\
\hline Case no 3 & $\begin{array}{l}\text { 1. Birth weight }<1.5 \mathrm{~kg}(\mathrm{VLBW}) \\
\text { 2. Culture positive sepsis } \\
\text { 3. Use of ototoxic medications for more than } 5 \text { days } \\
\text { 4. Stay in NICU for more than } 5 \text { days. ( Preterm }<33 \text { weeks ) }\end{array}$ \\
\hline Case no 4 & $\begin{array}{l}\text { 1. Culture positive sepsis } \\
\text { - Meningitis } \\
\text { 2. Use of ototoxic medications for more than } 5 \text { days } \\
\text { 3. TORCH infections. (CMV and Rubella) } \\
\text { 4. Stay in NICU for more than } 5 \text { days. (ICH) }\end{array}$ \\
\hline Case no 5 & $\begin{array}{l}\text { 1. Culture positive sepsis } \\
\text { 2. Use of ototoxic medications for more than } 5 \text { days. } \\
\text { 3. Stay in NICU for more than } 5 \text { days. }\end{array}$ \\
\hline
\end{tabular}

Gestational age and age of detection of hearing loss: Gestational age and age at which hearing screening was done is shown in Table 4. Mean gestational age was 38.33 weeks in all high risk neonates while it was 36.2 weeks in neonates with hearing loss. Mean age at which initial screening was done was 14.19 days in all high risk neonates while it was 18.2 days in neonates with hearing loss. Mean age at which hearing loss was confirmed was 65.8 days.

Table-4: Gestational age and Age at which hearing screening done.

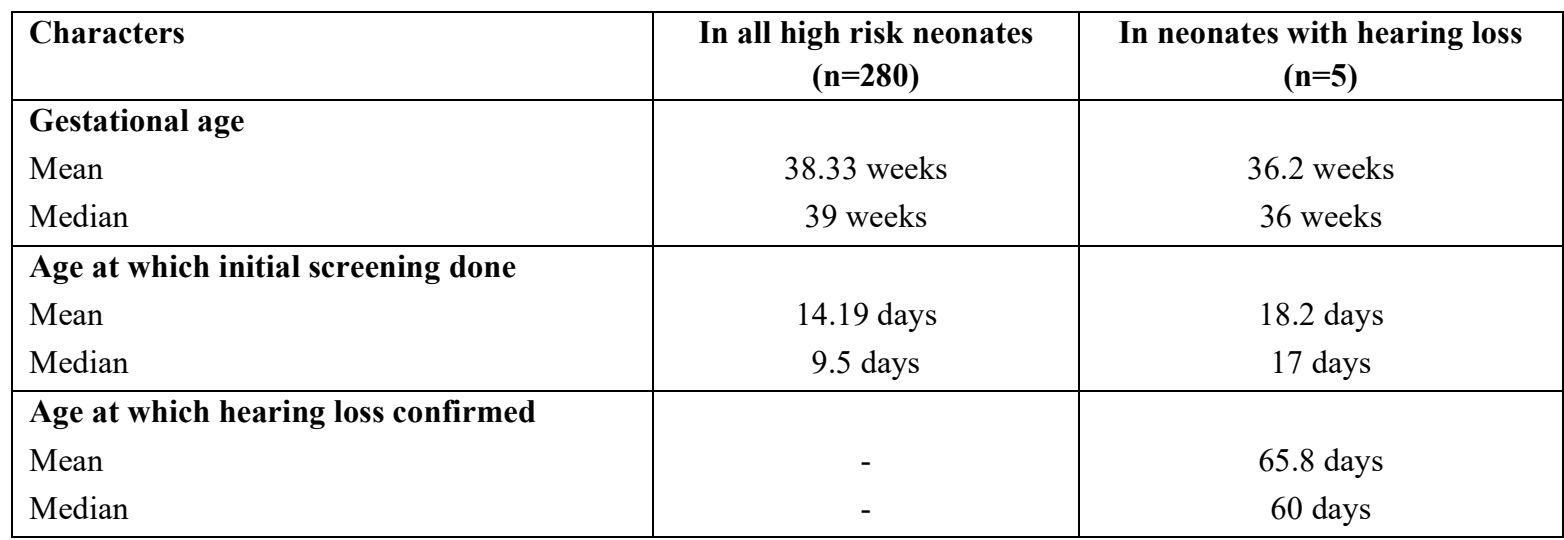




\section{Discussion}

Incidence of 17.8 per 1000 in the present study was higher than that in the studies by Nagapoornima $\mathrm{P}$ et al. (2002-2006) [3], Paul Abraham (2003-2009) [2] and Jewel John et al (2007-2009) [8] and was lower than that in the studies by Sudhir Rughani et al. (2010) [9] and Kumar et al (2010-2011) [10]. This is a very high incidence in relation to other congenital defects for which cure can be provided. The findings of this study correlates well with most of the large scale international studies $[11,12,13,14]$.

In the present study, hearing loss with individual risk factor in decreasing order of frequency was: 2 out of 4 $(50 \%)$ babies with meningitis had hearing loss. This finding was similar to the finding by Jewel John et al. (2007-2009) [8]. In the present study, 1 out of $2(50 \%)$ neonates with ICH had hearing loss.

Hearing loss in craniofacial anomalies was 1 out of 5 $(20 \%) .1$ out of $9(11.11 \%)$ babies with TORCH infections had hearing loss which was almost similar to the findings by Kumar et al. (2010-2011) [10]. 4 out of $45(8.88 \%)$ neonates with culture positive sepsis including bacterial meningitis had hearing loss which was lower than in the study by Liu Zhiqi et al. (20062008) [15].

Hearing loss in VLBW babies was 1 out of 14 (7.14\%) which was higher than in the study by Jowel John et al. (2007-2009) [8] and Taghdiri Mohammad Mehdi et al. (2005-2006) [16]. Hearing loss in preterm was 1 out of $23(4.34 \%)$. Hearing loss in severe birth asphyxia was 1 out of $25(4 \%)$ which was lower than in the study by Jewel John et al. (2007-2009) [8] and Kumar et al. (2010-2011) [10], but higher than in the study by Nagapoornima P et al. (2002-2006)[3].

In the present study no hearing loss was seen in babies with family history of childhood hearing loss, which was similar to the study by Jowel John et al. (20072009) [8] while not match the findings of the study by Nagapoornima P et al. (2002-2006) [3] Also no hearing loss was seen in babies with syndromes associated with hearing loss. In the present study, mean age at which initial hearing screening was done was 14.19 days and mean age at which hearing loss was confirmed was 65.8 days.

This figure matches the recommendation given by $\mathrm{JCIH}$ 2007 [6] position statement. JCIH 2007 position statement recommended that the hearing of all infants should be screened at no later than 1 month of age. Those who do not pass screening should have a comprehensive audiological evaluation at no later than 3 months of age [6].

\section{Conclusion}

This study shows that there is high incidence of hearing loss in at risk infants. In many parts of India hearing screening is still not being done. Though universal hearing screening is ideal, one can start off with screening the high risk neonates in whom hearing loss should not be missed. The consequences of not detecting hearing loss in early life and initiation of rehabilitative measures can have devastating implications for the child.

We recommend that paediatricians consider incorporating a basic hearing screen for all high risk newborns. In locations where getting infants to return for outpatient screening and testing is difficult, a substantially lower failure rate will likely be achieved by using both $\mathrm{OAE}$ and $\mathrm{ABR}$ at the same sitting.

\section{Funding: Nil, Conflict of interest: Nil} Permission from IRB: Yes

\section{References}

1. Haddad Joseph. Hearing loss. In: Kliegman RM, editor. Nelson Textbook of Pediatrics. Philadelphia: Elsevier; 2012. p. 2188-2196.

2. Paul Abraham K. Early Identification of Hearing Loss and Centralized Newborn Hearing Screening Facility-The Cochin Experience. Indian Pediatrics 2011; 48: 355-359.

3. Nagapoornima P, A Ramesh, Srilakshmi, Rao Suman, Patricia P L, Gore Madhuri et al. Universal Hearing Screening. Indian J Pediatr 2007; 74 (6) : 545-9.

4. Evidence based clinical practice guidelines. National Neonatology Forum, India. October 2010.p. 265-270.

5. Joint Committee on Infant hearing (1994) Position statement. ASHA 36:38-41.

6. Joint Committee on Infant Hearing Position Statement. Pediatrics 2007; 120 (4): 898-921. 
Original Research Article

7. Joint Committee on Infant Hearing (JCIH) Position Statement: Principles and Guidelines for Early Hearing Detection and Intervention Programs. Pediatrics 2000; 106 (4): 798-817.

8. Jewel John, Varghese P V, Singh Tejinder, Varghese Ashish. Newborn Hearing Screening-Experience at a Tertiary Hospital in Northwest India. International Journal of Otolaryngology and Head \& Neck Surgery 2013, 2: 211-214.

9. Sudhir Rughani, Bhadresh Vyas, Vikas Sinha, Maulik Shah, Yamini Kapileshwarkar, Sonal Shah. Hearing screening in new born (A cross-sectional study). World Articles in Ear, Nose and Throat 2011; Volume 4 (1). http: //waent. org/archives/2011/Vol41/20110515. Hearing Screening /otoacoustic-emissions. $\mathrm{htm}$ accessed on 16.11.2011.

10. Kumar Anil YC, Chandrashekar and Sodhi Kirandeep. Universal Hearing Screening In Newborn. International Journal of Basic and Applied Medical Sciences 2013; 3 (2): 116-121.

11. Flynn Mark, Austin Nicola, Flynn Traci Schmidtke, Ford Rodney, Buckland Liz. Universal Newborn Hearing Screening introduced to NICU infants in Canterbury Province, New Zealand. Journal of the New Zealand Medical Association 2004; Vol 117 No 1206.
12. Khaimook Wandee, Chayarpham Satit, Dissaneevate Supaporn. The High-Risk Neonatal Hearing Screening Program in Songklanagarind Hospital. J Med Assoc Thai 2008; 91 (7): 10381042.

13. Basar Figen, Gul Guven Ayse. The Distribution of Risk Factors among High Risk Infants Who Failed at Hearing Screening. The Mediterranean Journal of Otology 2008; 211-216.

14. Ur Rehman M, Mando K, Rahmani A, Imran A, Ur Rehman N, Gowda K, Chedid F. Screening for neonatal hearing loss in the Eastern region of United Arab Emirates. East Mediterr Health J. 2012 Dec;18 (12):1254-6.

15. Liu Z, Liu L. Hearing screening and diagnosis in a large sample of infants in Central China. J Med Screen. 2013 Mar;20(1):21-6. doi: 10.1177/0969141313478002. Epub 2013 May 13.

16. Taghdiri Mohammad Mehdi, Eghbalian Fatemeh, Emami Faranak, Abbasi Behnas, Zandevakili Hasan et al. Auditory Evaluation of High Risk Newborns by Automated Auditory Brain Stem Response. Iran J Pediatr. Dec 2008; 18 (4): 330-334.

\section{How to cite this article?}

Vaghasiya D, Deb S, Gogoi P.R, Nongrum H.B, Lurshay R. M. Hearing loss in high risk neonates. Int. J Pediatr Res. 2016;3(11):792-797.doi:10.17511/ijpr.2016.i11.03. 